\title{
Postoperative Complications in Thyroidectomy Performed at Hospital Escuela Universitario and Hospital General San Felipe, Tegucigalpa MDC in 2018
}

ISSN: 2578-0379

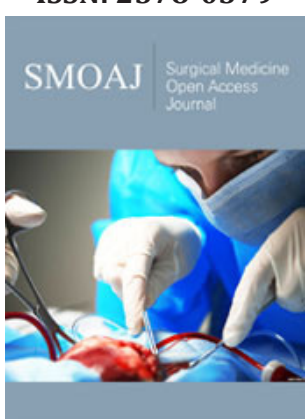

*Corresponding author: Rosales FES, Department of Surgeon General, USA

Submission: 海 July 14, 2021

Published: 䟧 August 18, 2021

Volume 4 - Issue 3

How to cite this article: Rosales FES, Bueso HES. Postoperative Complications in Thyroidectomy Performed at Hospital Escuela Universitario and Hospital General San Felipe, Tegucigalpa MDC in 2018. Surg Med Open Acc J. 4(3). SMOAJ.000590. 2021 DOI: 10.31031/SMOAJ.2021.04.000590

Copyright@ Rosales FES, This article is distributed under the terms of the Creative Commons Attribution 4.0 International License, which permits unrestricted use and redistribution provided that the original author and source are credited.

\author{
Rosales FES* and Bueso HES \\ Department of Surgeon General, USA
}

\begin{abstract}
Introduction: Specific complications of thyroid surgery include injury to the recurrent laryngeal nerve and the parathyroid glands, without being exempt from other complications such as infection of the surgical site, bleeding, surgical wound dehiscence, and others.
\end{abstract}

Methods: The present study is retrospective observational and aims to analyze the condition of thyroid surgery in the country, specifically in state hospitals in Tegucigalpa, its rate of complications and its relationship with associated factors before the procedure.

Result: A total of 92 patients undergoing thyroid surgery were studied at the Hospital Escuela and Hospital General San Felipe by reviewing the clinical record. The preoperative status, comorbidities and the surgical procedure performed were recorded and were related to the main complications mentioned above.

Conclusion: A global rate of complications related to laryngeal nerve injury of $15 \%$ and late hypocalcemia of $7 \%$ was found, which are higher than the incidences reported in international series, however these vary from $0 \%$ of recurrent laryngeal nerve injury in thyroidectomies subtotal up to $22 \%$ in radical dissections in patients with neo proliferative processes.

Keywords: Hypocalcemia; Parathyroid; Surgical complications; Thyroid; Thyroidectomy

\section{Introduction}

Postoperative complications in thyroid surgery constitute an important cause of morbidity in patients undergoing these procedures. Although the complication rates in international series are low, the associated morbidity and hospital costs justify their study [1]. Recurrent laryngeal nerve injuries and symptomatic hypocalcemia are the main complications inherent to thyroid surgery and these are associated with multiple factors specific to the patient and their pathology, as well as events related to the surgical procedure. In Honduras there are few studies related to thyroid disease and none studying their specific postoperative complications. The purpose of this study is to provide information on the status of endocrine surgery in the country and specifically on thyroid surgery and relate it to the global rates of postoperative complications [2]. Having an initial diagnosis will provide the option to planning and management of efforts to reduce the rate of postoperative complications in thyroid surgery. This study provides useful information for administrative decision making, implementation of management policies and protocols, benefiting the population served, and the institutions involved in an attempt to identify predisposing characteristics of postoperative complications [3].

\section{Methodology}

A review of the clinical records of patients who had undergone thyroid surgery was carried out. Preoperative studies were compiled establishing thyroid function status and calcium metabolism, determining levels of thyroid hormones, albumin, calcium, vitamin D, thyroglobulin, albumin, thyroglobulin and levels of parathyroid hormone. Vocal cord function was verified by preoperative nasofibroscopy. The existence of comorbidities and associated 
conditions such as the presence of goiter, and other associated pathologies were verified. The suspicion or diagnosis of thyroid cancer was determined through the use of ultrasonography, fine needle aspiration biopsy or other diagnostic techniques such as CT, scintigraphy and or magnetic resonance imaging [4-7].

A review was made of the surgical indication, the surgical technique used, the operative findings, the visualization and identification of structures of interest, intraoperative complications and the biopsy result. The control measurements of thyroid metabolism, calcium and the existence or not of complications typical of the procedure in the immediate postoperative period and the control carried out in the outpatient clinic were verified [8].

Symptomatic hypocalcemia was identified using serum calcium reference values according to the laboratory used associated with hypocalcemia symptoms such as tetany, cramps and / or paresthesia and the need for intravenous calcium replacement administration. Laryngeal nerve injury was identified with recurrent laryngeal nerve palsy with its clinical manifestations. General data information, admission diagnosis, procedure performed, postoperative evolution notes and subsequent evaluations in the consultation are essential for data processing [9-11].

\section{Result}

A total of 92 cases of patients who underwent thyroidectomy in 2018 were studied, 30 at Hospital Escuela and 62 at Hospital General San Felipe. In this study, 95\% of the patients were women, while $5 \%$ were male. More than a third of the patients were older than 60 years (Table 1); [12].

Regarding the origin, $71.43 \%$ of the cases lived in an urban area, and $28.57 \%$ of the cases studied came from rural areas of the country. Regarding the personal pathological antecedents found in the patients of this study. Hypertension was present in $42 \%$ of the study participants, followed by obesity and diabetes mellitus with $25 \%$ and $18 \%$ respectively [13]. Note that because there are patients with multiple comorbidities, the sum of all the percentages exceeds $100 \%$. Tables [2-4] show the complication index, of transient and permanent hypocalcemia, and recurrent laryngeal nerve injury, respectively. The distribution by admission diagnoses was made and a comparison was made with the definitive postoperative histological diagnosis [14]. It is observed that $11 \%$ of the patients with negative pathology presented mediate hypocalcemia while $30 \%$ of the patients with positive pathology due to malignancy presented transient hypocalcemia. $14 \%$ of the patients with a positive biopsy result for malignancy presented permanent hypocalcemia. Only $4 \%$ of the patients undergoing thyroidectomy with a negative pathological anatomy result had some type of recurrent laryngeal nerve injury (Figure 1); [15-18]. Tables 5 \& 6 show the incidence of transient, permanent hypocalcemia and recurrent laryngeal nerve injury according to the surgical procedure performed [19]. It can be seen that subtotal thyroidectomy had $0 \%$ complications, total thyroidectomy had $20 \%$ and $6 \%$ transient and permanent hypocalcemia respectively. Total thyroidectomy has $15 \%$ recurrent laryngeal nerve injury, while when radical dissection was performed, $21 \%$ of patients developed unilateral or bilateral recurrent laryngeal nerve injury [20].

Table 1: Age distribution in post-thyroidectomy patients at Hospital General San Felipe and Hospital Escuela in 2018. N: 92.

\begin{tabular}{|c|c|c|}
\hline Age & Frequency & Percentage \\
\hline $18-29$ & 10 & $10.87 \%$ \\
\hline $30-39$ & twenty-one & $22.83 \%$ \\
\hline $40-49$ & 14 & $15.22 \%$ \\
\hline $50-59$ & 14 & $15.22 \%$ \\
\hline 60 or older & 33 & $35.87 \%$ \\
\hline Total & 92 & $100.00 \%$ \\
\hline
\end{tabular}

Table 2: Transient hypocalcemia according to admission diagnosis in post-operated thyroidectomy patients at Hospital General San Felipe and Hospital Escuela in 2018. N: 92.

\begin{tabular}{|c|c|c|c|}
\hline \multicolumn{4}{|c|}{ Mediate Hypocalcemia According to Admission Diagnosis } \\
\hline \multicolumn{4}{|c|}{ Negative pathological anatomy due to malignancy } \\
\hline Admission diagnosis & Yes & Not & Total \\
\hline Multinodular goiter & $3(16 \%)$ & $15(83 \%)$ & 18 \\
\hline Thyroid cancer & 0 & $6(100 \%)$ & 6 \\
\hline Thyroid nodule & $2(11 \%)$ & $16(88 \%)$ & 18 \\
\hline Total & $5(11 \%)$ & $37(88 \%)$ & 42 \\
\hline \multicolumn{4}{|c|}{ Positive pathological anatomy due to malignancy } \\
\hline Admission diagnosis & Yes & Not & Total \\
\hline Multinodular goiter & $1(20 \%)$ & $4(80 \%)$ & 5 \\
\hline Thyroid cancer & $11(30 \%)$ & $25(69 \%)$ & 36 \\
\hline Thyroid nodule & $3(33 \%)$ & $6(66 \%)$ & 9 \\
\hline Total & $15(30 \%)$ & $35(70 \%)$ & 50 \\
\hline
\end{tabular}


Table 3: Permanent hypocalcemia according to admission diagnosis in post-operated thyroidectomy patients at Hospital General San Felipe and Hospital Escuela in 2018. N: 92.

\begin{tabular}{|c|c|c|c|}
\hline \multicolumn{4}{|c|}{ Delayed Hypocalcemia According to Admission Diagnosis } \\
\hline \multicolumn{4}{|c|}{ Negative pathological anatomy due to malignancy } \\
\hline Admission diagnosis & Yes & Not & Total \\
\hline Multinodular goiter & 0 & $18(100 \%)$ & 18 \\
\hline Thyroid cancer & 0 & $6(100 \%)$ & 6 \\
\hline Thyroid nodule & 0 & $18(100 \%)$ & 18 \\
\hline Total & 0 & 42 & 42 \\
\hline \multicolumn{4}{|c|}{ Positive pathological anatomy due to malignancy } \\
\hline Admission diagnosis & Yes & Not & Total \\
\hline Multinodular goiter & $1(20 \%)$ & $4(80 \%)$ & 5 \\
\hline Thyroid cancer & $3(8 \%)$ & $33(91 \%)$ & 36 \\
\hline Thyroid nodule & $3(33 \%)$ & $6(66 \%)$ & 9 \\
\hline Total & $7(14 \%)$ & $43(86 \%)$ & 50 \\
\hline
\end{tabular}

Table 4: Laryngeal nerve injury according to admission diagnosis in post-operated thyroidectomy patients at Hospital General San Felipe and Hospital Escuela in 2018. N: 92.

\begin{tabular}{|c|c|c|c|c|c|}
\hline \multicolumn{6}{|c|}{ Laryngeal Nerve Injury According to Admission Diagnosis } \\
\hline \multicolumn{6}{|c|}{ Negative pathological anatomy due to malignancy } \\
\hline Admission diagnosis & No Injury & Bilateral & Right & Left & Total \\
\hline Multinodular goiter & $15(84 \%)$ & fifteen $(\%)$ & fifteen $(\%)$ & fifteen $(\%)$ & 18 \\
\hline Thyroid cancer & $6(100 \%)$ & 0 & 0 & 0 & 6 \\
\hline Thyroid nodule & $18(100 \%)$ & 0 & 0 & 0 & 18 \\
\hline Total & $39(92 \%)$ & $1(2 \%)$ & $1(2 \%)$ & $1(2 \%)$ & 42 \\
\hline \multicolumn{6}{|c|}{ Positive pathological anatomy due to malignancy } \\
\hline Admission diagnosis & Normal & Bilateral & Right & Left & Total \\
\hline Multinodular goiter & $1(80 \%)$ & 0 & 0 & $1(20 \%)$ & 5 \\
\hline Thyroid cancer & $28(77 \%)$ & $1(3 \%)$ & $2(5 \%)$ & $5(14 \%)$ & 36 \\
\hline Thyroid nodule & $7(77 \%)$ & 0 & 0 & $2(22 \%)$ & 9 \\
\hline Total & $39(78 \%)$ & $1(2 \%)$ & $2(4 \%)$ & $8(16 \%)$ & 50 \\
\hline
\end{tabular}

Table 5: Hypocalcemia according to procedure performed in post thyroidectomy patients at Hospital General San Felipe and Hospital Escuela in 2018. N: 92.

\begin{tabular}{|c|c|c|c|c|}
\hline \multirow{2}{*}{ Process } & \multicolumn{2}{|c|}{ Typocalcemia According to the Procedure Performed } \\
\cline { 2 - 5 } & Yes & Not & \multicolumn{2}{|c|}{ Permanent hypocalcemia } \\
\hline Subtotal Thyroidectomy & 0 & $11(100 \%)$ & 0 & $11(100 \%)$ \\
\hline Total Thyroidectomy & $13(20 \%)$ & $51(80 \%)$ & $4(6 \%)$ & $60(94 \%)$ \\
\hline Radical dissection & $7(41 \%)$ & $10(59 \%)$ & $3(18 \%)$ & $14(82 \%)$ \\
\hline Total & $20(22 \%)$ & $72(78 \%)$ & $7(7 \%)$ & $85(93 \%)$ \\
\hline
\end{tabular}

Table 6: Recurrent laryngeal nerve injury according to Procedure Performed in post-operated thyroidectomy patients at Hospital General San Felipe and Hospital Escuela in 2018. N: 92.

\begin{tabular}{|c|c|c|c|c|c|}
\hline \multicolumn{7}{|c|}{ Recurrent Laryngeal Nerve Injury According to Procedure Performed } \\
\hline Process & Normal & Bilateral & Right & Left & Total \\
\hline Subtotal thyroidectomy & $11(100 \%)$ & 0 & 0 & 17 \\
\hline Total thyroidectomy & $54(84 \%)$ & $1(2 \%)$ & $2.3 \%)$ & $7(11 \%)$ & eleven \\
\hline Radical dissection & $13(76 \%)$ & fifteen (\%) & fifteen (\%) & $2(11 \%)$ & 64 \\
\hline Total & $78(85 \%)$ & $2(2 \%)$ & $3(3 \%)$ & $9(10 \%)$ & 92 \\
\hline
\end{tabular}




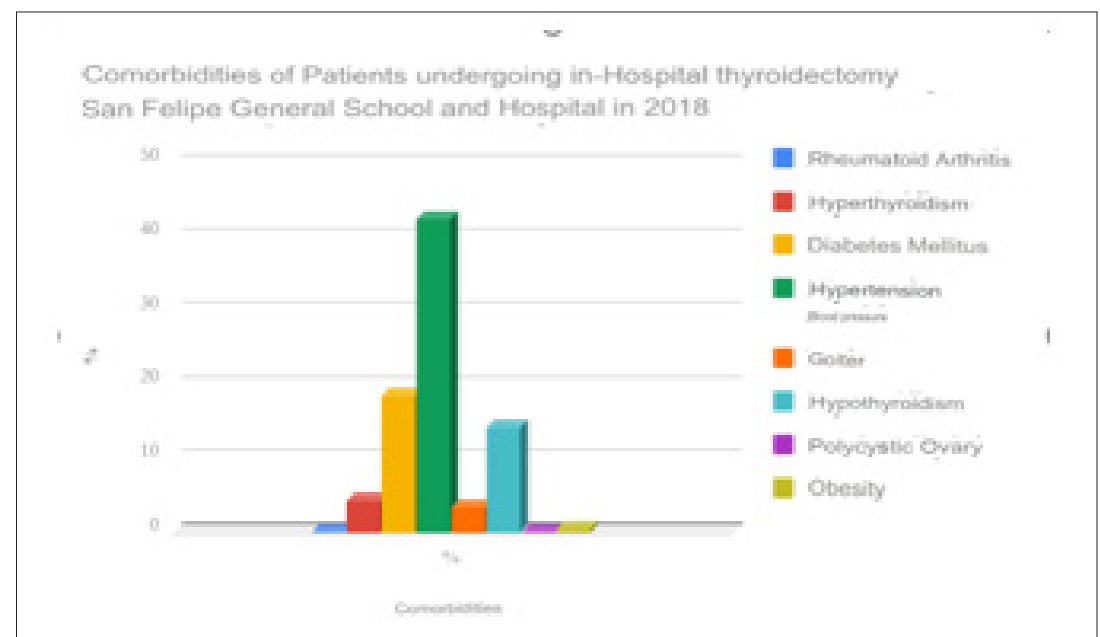

Figure 1: Comorbidities of patients undergoing thyroidectomy at Hospital Escuela and Hospital General San Felipe in 2018.

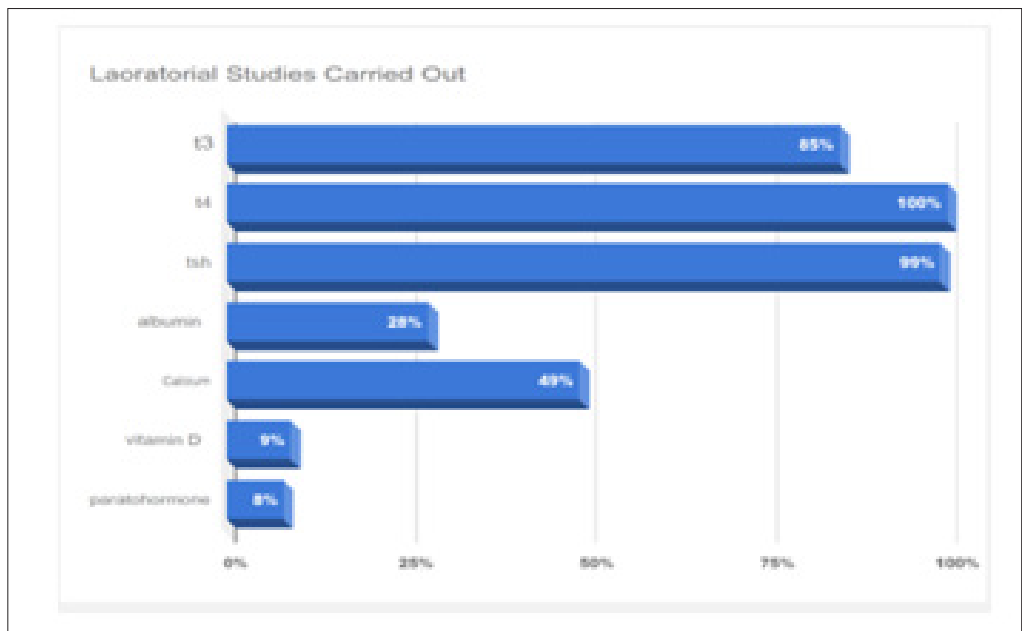

Figure 2: Laboratory studies performed in post-operative thyroidectomy patients at Hospital General San Felipe and Hospital Escuela in 2018. N: 92.

A significant percentage of the laboratory studies under study were not performed or were not recorded in the clinical record. Figure 2 shows that only T4 was performed in $100 \%$ of the cases and that Albumin, Calcium, Vitamin D and Parathyroid hormone were not performed in more than $50 \%$ of the cases. Similarly, preoperative and postoperative laryngoscopy was performed only in $1 \%$ and $24 \%$ respectively, thus making a complete analysis of the collected data impossible [21-23].

\section{Discussion}

In the present study, 92 clinical records of patients undergoing thyroid surgery at Hospital Escuela and Hospital General San Felipe in 2018 were analyzed. The indications for surgery were multiple, including thyroid nodules, goiter, cancer, among others, and the procedures varied according to the etiology ranging from subtotal thyroidectomies to radical neck dissections [24].

The sociodemographic distribution has a marked inclination since the majority of patients studied are women in $98.39 \%$ compared to the male sex, representing only $1.61 \%$ of the total sample. The age distribution shows that the patients treated for thyroid disease are, for the most part, elderly, since $50 \%$ of the sample is over 50 years of age. $71 \%$ of the patients studied came from urban areas. The profile of a patient undergoing thyroid surgery at the Hospital Escuela y Hospital General San Felipe is an elderly woman from an urban area.

Comorbid states have a decisive influence on the diagnosis and treatment of thyroid disease, be it medical or surgical. In our study, the main pathologies found were recorded in which arterial hypertension was predominant, since $58.06 \%$ of the patients in the study were hypertensive, diabetes mellitus is found in $18 \%$ of patients. These, although it is true, do not directly affect the thyroid pathology, they condition and limit the surgical action since it increases the index of trans and postoperative bleeding as well as alters the subsequent inflammatory process. It was found that $3 \%$ of the sample had hyperthyroidism and hypothyroidism in 14.52 [25]. 
The main complications were related to the admission diagnosis of each patient, and later it was separated into 2 groups. The first was the one in which, regardless of their admission diagnosis, they obtained a negative pathological study for malignancy, and the second group those in which the pathological study was positive for malignancy. The first data that is pertinent to note is that not all cases admitted with a diagnosis of thyroid cancer have positive biopsies for malignancy in the postoperative period. Of the patients admitted with a diagnosis of thyroid cancer, 6 obtained a negative biopsy due to malignancy, these cases represent a false positive.

Hypocalcemia is a complication that can occur mediate and be temporary in most cases or be permanent. It is said that it is permanent hypocalcemia when there is serum hypocalcemia associated with its symptoms, it is generally worth calcium intake. The patients who were admitted with a diagnosis of multinodular goiter were the ones who showed the most hypocalcemia in a mediate and transitory way (16\%), since it was not necessary to continue the therapy for more than 6 months in the follow-up visits. None of the patients with a diagnosis of thyroid cancer on admission and negative postoperative biopsies showed transient hypocalcemia [26,27].

Regarding permanent hypocalcemia, none of the patients with negative postoperative biopsies showed permanent hypocalcemia, even those admitted with a diagnosis of thyroid cancer 7 of the patients studied showed permanent hypocalcemia, all of them with postoperative biopsies positive for malignancy, this represents $7 \%$ of the total sample and $14 \%$ of the patients with postoperative biopsies positive for malignancy [28].

Hypocalcemia can manifest as a complication due to the complexity of the surgical procedure or due to a distorted anatomy. This is reflected in the data obtained, since none of the patients who underwent subtotal thyroidectomy suffered from hypocalcemia, only $6 \%$ of those who underwent total thyroidectomy, and $18 \%$ of the patients who underwent radical dissection had permanent hypocalcemia. Likewise, we observed that no injury to the RLN was found in the subtotal thyroidectomies, however, $16 \%$ of the RLN injury was observed in the total thyroidectomies and $24 \%$ of the radical dissections, which means that 1 in 4 patients underwent Radical neck dissection due to surgical pathology presents a degree of injury to the RLN. It was also shown that bilateral RLN lesions are more frequent in neck dissection, with $5 \%$ in dissections vs $2 \%$ in total thyroidectomies. This shows the highest complication rate according to the complexity of the procedure [29].

Patients with a positive pathological anatomy due to malignancy, it should be noted that this procedure prevailed in all surgical interventions in $80.65 \%$ of the cases, $5 \%$ of the cases with admission diagnosis of thyroid cancer and a confirmatory diagnosis of malignancy presented bilateral paralysis. of the vocal cords, in addition $5 \%$ of the cases presented paralysis of the left vocal cord, with regard to thyroid nodule, the absence of post-surgical laryngoscopy prevailed in $77.78 \%$ and $22.22 \%$ presented paralysis of the left vocal cord, already taking the issue of multinodular goiter, $50 \%$ of the cases presented paralysis of the left vocal cord and $50 \%$ were reported as normal.

It was evidenced that the patients under study were not carried out with the preoperative study profile recommended by the international literature. One of the most alarming indices is the percentage of laryngoscopies performed, since they were performed only in $1 \%$ of the cases evaluated. Likewise, it was observed that the levels of parathyroid hormone, calcium, vitamin $\mathrm{D}$ and thyroglobulin were not adequately evaluated in the preoperative period. This is relevant since, as it does not have a preoperative parameter, it will not be possible to discern between a postoperative complication and an alteration in calcium metabolism or in RLN dysfunction prior to the surgical procedure. Also, having a complete preoperative profile could easily identify patients at higher risk of postoperative complications [30]. It is clear that the difficulty in completing the ideal preoperative studies has a multifactorial origin, but the economic factor plays a large role in this. Both the Hospital Escuela and the Hospital General San Felipe are state institutions with limited financial resources and the patients treated in these centers are, for the most part, incapable of performing studies such as laryngoscopies, tomographies, and special tests in private centers.

\section{Conclusion}

The profile of a patient undergoing thyroid surgery at the Hospital Escuela y Hospital General San Felipe is an elderly female patient from an urban area, which predisposes the presence of associated pathologies and comorbid conditions that condition the presentation and evolution of the disease. surgical thyroid disease.

The main comorbidities present in patients undergoing thyroidectomy are hypertension, obesity, and diabetes mellitus. Of the total of patients studied, $20 \%$ presented transient hypocalcemia, $7 \%$ presented permanent hypocalcemia and $15 \%$ presented a degree of recurrent laryngeal nerve injury. The patients most at risk of presenting postoperative complications in thyroidectomy are those with malignant neoplastic pathology in whom radical neck dissection is performed [31].

\section{Gratitude}

Thanks to our parents, for being the main promoters of our dreams, for trusting and believing in our expectations, for the advice, values and principles that they have instilled in us.

\section{References}

1. Thyroid gland surgery josé luis pardal-refoyo sacyl. Zamora Healthcare Complex. Otolaryngology Service. Zamora, Spain.

2. Sosa MG, Ernand RS (2016) Thyroidectomy at "Calixto García" general hospital. Cuban Journal of Surgery.

3. Pizarro FI (2013) Thyroid and goiter: Historical evolution and its great characters default. Rev Medica Condes.

4. (2017) Sociodemographic and epidemiological characterization of the most frequent neoplasms attended at Hospital Escuela Universitario, Honduras. Champion Federico Mendoza Talavera 13(1): 1.

5. Vasquez SP, Mendoza RZ (2003) Fine needle aspiration biopsy of the thyroid in the teaching hospital: Evaluation of its diagnostic accuracy as a screening test. Rev Med Post Unah 8(25). 
6. Eduardo Núñez, Luis Munguía. Surgical approach of thyroid nodules with follicular lesion of undetermined significance. Surgical approach to thyroid nodules with follicular lesion of undetermined significance.

7. Flores OR (1992) Combined surgery in thyroid cancer. Honduran Medical Journal 60.

8. Paguada DAO (1987) Surgical thyroid pathology, incidence, treatment modality and complications at leonardo hospital martinez valenzuela in the period from 1981-1987.

9. Fernández M (2015) Pathology and surgery of the thyroid and parathyroid glands official presentation of the Spanish society of otorhinolaryngology and cervico-facial pathology 2015.

10. Skandalakis J (2015) Surgery foundations of surgical anatomy 2015.

11. Sanabria A, Chala A, Ramírez A, Álvarez A (2014) Cervical surgical anatomy of importance in thyroid surgery. Rev Colomb Cir 29: 50-58.

12. Henry BM, Sanna B, Vikse J, Graves MJ, Spulber A, et al. (2017) Zuckerkandl's tubercle and its relationship to the recurrent laryngeal nerve: A cadaveric dissection and meta-analysis. Auris Nasus Larynx 44(6): 639-647.

13. Rojas M, Quijano Y, Miguel R, Bernal L (2016) Anatomical variations of the recurrent laryngeal nerve in a Colombian population sample recurrent laryngeal nerve in a sample of the Colombian population. Rev Fac Med 64(2): 207-213.

14. Toda FILL, Salas MMV (2017) Thyroid pathology update. In: Aepap (Edr.), Pediatrics Update Course pp. 161-174.

15. Fernandez M (2015) Official presentation of the Spanish society of otorhinolaryngology and cervico-facial pathology 2015. Pathology and Surgery of the Thyroid and Parathyroid Glands.

16. Garcia G (2016) Thyroid physiology. Med Int Mex 32(5): 569-575.

17. Ortega PR, Urra AB, Compan AJ (2011) Clinical experience in total thyroidectomy. Rev Otorrinolaringol Cir Head Neck 71: 53-56.

18. Joliat GR, Guarnero V, Demartines AN, Schweizer V, Matter M (2017) Recurrent laryngeal nerve injury after thyroid and parathyroid surgery incidence and postoperative evolution assessment. Medicine 96(17): e6674.

19. Jiang Y, Gao B, Zhang X, Zhao J, Chen J, et al. (2014) Prevention and treatment of recurrent laryngeal nerve injury in thyroid surgery. Int J Clin Exp Med 7(1): 101-107.
20. García JA, Calderón J, Zarza VV, Velarde MC, Rodríguez MD. The neuromonitoring in cervical endocrine surgery. Detection and Intraoperative Prevention of Laryngeal Paralysis.

21. Jeannon JP, Orabi JP, Bruch GA, Abdalsalam HA, Simo R (2009) Diagnosis of recurrent laryngeal nerve palsy after thyroidectomy: A systematic review. Int J Clin Pract 63(4): 624-629.

22. Bello AM, Real PR, García MAR (2014) Postoperative morbidity in patients undergoing thyroidectomy at the general hospital of Acapulco. Three years of experience. Elsevier Cir Gen 3(2): 91-95.

23. Paek SH, Kang KH, Park SJ (2018) A comparison of robotic versus open thyroidectomy for papillary thyroid cancer. Surg Laparosc Endosc Percutan.

24. Thyroidectomy without cervical incision by endoscopic approach biaxyl-biareolar. First impressions after its introduction in a specialized unit. Literature Review. Mount Sinai Hospital, New York, Ny, USA.

25. Gordillo VR, Vásquez WI, Andrade CA (2017) Thyroidectomy transoral endoscopic by vestibular approach (Toetva): Report of the first human case in Latin America. Hospital del Instituto Ecuatoriano de Seguridad Social (Iess), Ibarra, Ecuador. Rev Chil Cir 69(1): 60-64.

26. Tae K, Ji YB, Cho SH, Lee SH, Kim DS, et al. (2012) Early surgical outcomes of robotic thyroidectomy by a gasless unilateral axillo-breast or axillary approach for papillary thyroid carcinoma: 2 years' experience. Head Neck 34(5): 617-625.

27. Benhidjeb T, Wilhelm T, Harlaar J, Kleinrensink GJ, Schneider TAJ, et al. (2009) Natural orifice surgery on thyroid gland: Totally transoral videoassisted thyroidectomy (Tovat): Report of first experimental results of a new surgical method. Surg Endosc 23(5): 1119-1120.

28. Refoyo JLP (2012) Utility of neuromonitoring in thyroid surgery. Acta Otorrinolaringol Esp 63(5): 355-363.

29. Nuño P, Montañez JB, Carrasco CR, Negrín AP (2002) Immediate postoperative period in patients undergoing total thyroidectomy in ICU. Enferm Intensiva 13(2): 78-84.

30. Edafe O, Antakia R, Laskar N, Uttley L, Balasubramanian SP (2014) Systematic review and meta-analysis of predictors of post-thyroidectomy hypocalcemia. Br J Surg 101(4): 307-320.

31. Espino CP, Já RB, Caselles AM, González SJ, Pavía GA, et al. (2018) Use of the value of intact parathyroid hormone on the first postoperative day after total thyroidectomy as a predictor of permanent hypoparathyroidism. Endocrinol Diabetes Nutr. 